\title{
Fundamental Study on the Evaluation of Original the Vascular Lumen after Carotid Artery Stenting Using 3D-rotational Angiography with Diluted Contrast Medium
}

Shogo Yoda, ${ }^{1}$ Yusuke Hamada, ${ }^{1}$ Satoru Kawauchi, ${ }^{1}$ Hideyuki Sakuma, ${ }^{1}$ Tatsuo Amano, ${ }^{2}$ Masayuki Sato, ${ }^{3}$ and Yuji Matsumaru ${ }^{4}$

Objective: Due to the recent increase in the availability of cone-beam CT (CBCT), delineation of blood vessels and intracranial stents using CBCT has been well-reported, but reports using 3D-rotational angiography (3D-RA) have been few. We evaluated delineation of carotid artery stents using 3D-RA with diluted contrast medium.

Methods: We prepared simulated blood vessel phantoms covered by carotid artery stents different in material and shape. The phantoms were encapsulated with different concentration of contrast medium, and scanned using 3D-RA. The appropriate concentration of contrast medium was evaluated.

Results: The appropriate concentrations of diluted contrast medium were $50-17 \%$ for the Carotid Wall Stent (Boston Scientific, Natick, MA, USA) and 20\%-10\% for PRECISE (Johnson \& Johnson, Miami, FL, USA) and PROTÉGÉ (Covidien, Irvine, CA, USA).

Conclusion: The appropriate concentration of contrast medium varied with carotid artery stent. By selecting an appropriate degree of dilution, the stent shape, plaque, intimal thickening, and vascular lumen in the stent can be visualized. Therefore, it is possible to evaluate vascular lumen after carotid artery stenting.

Keywords > 3D-rotational angiography, carotid artery stenting, diluted contrast agent

\section{Introduction}

Recently, with the increased availability of angiographic systems mounted with the flat panel detector, the quality of images obtained by rotational imaging techniques such as cone-beam $\mathrm{CT}(\mathrm{CBCT})$ and 3D-rotational angiography

${ }^{1}$ Department of Radiological Technology, Toranomon Hospital, Tokyo, Japan

${ }^{2}$ Stroke Center, Kyorin University Hospital, Tokyo, Japan

${ }^{3}$ Cranial Nerve Surgery, National Hospital Organization Mito

Medical Center, Higashiibaraki, Ibaraki, Japan

${ }^{4}$ Division for Stroke, Department of Neurosurgery, Faculty of

Medicine, University of Tsukuba, Tsukuba, Ibaraki, Japan

Received: March 7, 2017; Accepted: August 4, 2017

Corresponding author: Shogo Yoda. Department of Radiological Technology, Toranomon Hospital, 2-2-2 Toranomon, Minatoku, Tokyo 105-8470, Japan

Email: shogoyoda@hotmail.co.jp

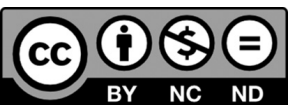

This work is licensed under a Creative Commons Attribution-NonCommercialNoDerivatives International License.

(C)2018 The Japanese Society for Neuroendovascular Therapy
(3D-RA) has markedly improved, and the range of application of these techniques has widened.

After carotid artery stenting (CAS), it is essential to evaluate the carotid stent (CS) shape, stent apposition to the vessel, and plaque protrusion into the vessel, and intravascular ultrasound (IVUS) is frequently used for this purpose. Delineation of intracranial stents using high-resolution CBCT (HRCBCT) and diluted contrast medium has been reported. ${ }^{1,2)}$ Benndorf et al. ${ }^{3)}$ also reported the usefulness of delineation of the CS and vascular wall by non-contrastenhanced CBCT. In addition, Hosokawa et al. ${ }^{4)}$ reported delineation of the CS using a diluted contrast medium.

At our institution, the angiographic system is equipped with CBCT, HRCBCT, and 3D-RA for rotational imaging. The imaging conditions are clearly separated, and we have encountered cases of insufficient resolution by CBCT and difficulty in evaluation due to movements and metal artifact by HRCBCT.

According to the literature, the appropriate degree of contrast dilution is the same for delineation of CS with different in material and structure (Carotid Wall Stent; 
Table 1 Differences in scanning conditions between 3D-RA and HRCBCT

\begin{tabular}{lcc} 
& 3D-RA & HRCBCT \\
Tube voltage $(\mathrm{kV})$ & $50-125$ (auto) & 80 \\
Exposure time $(\mathrm{sec})$ & 4 & 20 \\
Frame rate (fr/sec) & 30 & 30 \\
FPD size (inch) & 8 & 8 \\
Matrix & 1024 & 1024 \\
Pixel size $(\mu \mathrm{m})$ & 154 & 154 \\
Filter & $0.1 \mathrm{~mm} \mathrm{Cu}+1 \mathrm{~mm} \mathrm{Al}$ & None \\
Rotation angle (deg) & 210 & 210 \\
Projection images & 121 & 620 \\
Binning & $1 \times 1^{*}$ & $1 \times 1$ \\
\hline
\end{tabular}

"In 3D-RA, binning is $1 \times 1$ when the FPD size is 8 inches or less. HRCBCT: high-resolution cone beam CT; FPD: flat panel detector; 3D-RA: 3D rotational angiography

Boston Scientific, Natick, MA, USA, PRECISE; Johnson \& Johnson, Miami, FL, USA). In addition, individual differences in the common carotid artery blood flow are clinically disregarded.

In this study, by 3D-RA using diluted contrast medium, we visualized three elements, that is, shape of the CS, plaque and intimal thickening, and vascular lumen of the $\mathrm{CS}$, in one image. Also, as a basic study, we evaluated the degree of dilution appropriate for each CS by changing the degree of dilution of the contrast medium for CS different in structure and material. On the basis of the results, we retrospectively reviewed our past cases and evaluated an imaging technique that can be applied clinically to individual patients.

The objective of this study was to evaluate the optimal imaging conditions of 3D-RA with the adjustment of the degree of contrast medium dilution according to the CS type and individual difference in the carotid artery blood flow.

\section{Materials and Methods}

\section{Instruments}

The angiographic system used was an Allura XperFD20/20 (Philips Healthcare, Best, The Netherlands), and the workstation was Xtravision, which accompanied the system. The conditions of 3D-RA were as follows: tube voltage: automatically set, irradiation field size: 8 inch, and scanning time: 4.1 seconds (Table 1).

Three different stents were used for the evaluation: 1) Carotid Wall Stent, 2) PRECISE, and 3) PROTÉGÉ (Covidien, Irvine, CA, USA). As original phantoms, simulated blood vessels were prepared in which a simulated plaque (silicone-modified acrylic resin with a $\mathrm{CT}$ value of 50 Hounsfield units) was placed (Fig. 1). To measure the pixel values of the images obtained, the image processing software ImageJ (U.S. National Institutes of Health, Bethesda, MD, USA) was used.

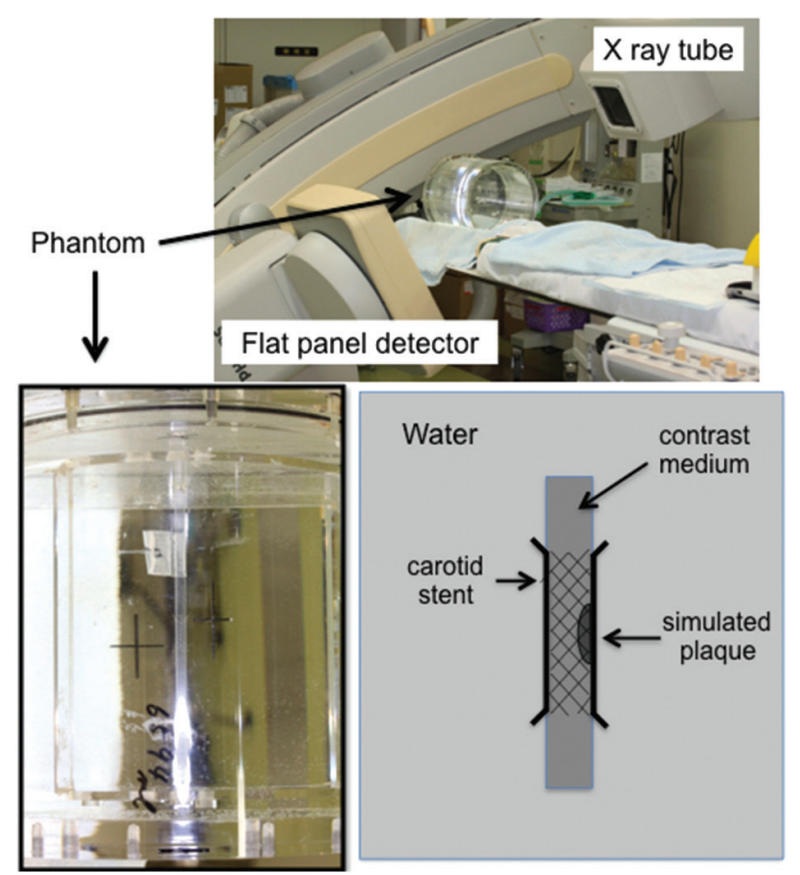

Fig. 1 Phantom images of various stents at different contrast dilution ratios.

\section{Phantom study to determine the effective contrast dilution ratio}

A contrast medium diluted at various degrees was encapsulated in original phantoms. Iopamidol 300 (300 mg iodine/ $\mathrm{mL}$, Bayer Yakuhin, Ltd., Osaka, Japan) diluted with physiologic saline to seven levels $(50 \%, 33 \%, 20 \%, 17 \%, 10 \%$, and $8 \%$ relative to the original preparation) was placed in the center of acrylic columnar water-filled phantoms $25 \mathrm{~cm}$ in diameter and imaged by 3D-RA (Fig. 1).

The image of each CS at each degree of dilution was reconstituted under the following conditions: cube size: $50 \%$, matrix: $512 \times 512 \times 512$ reconstruction, and reconstruction filter: very sharp. The image was displayed by partial maximum intensity projection (MIP) in multiplanar reconstruction (MPR) images, and the displayed 


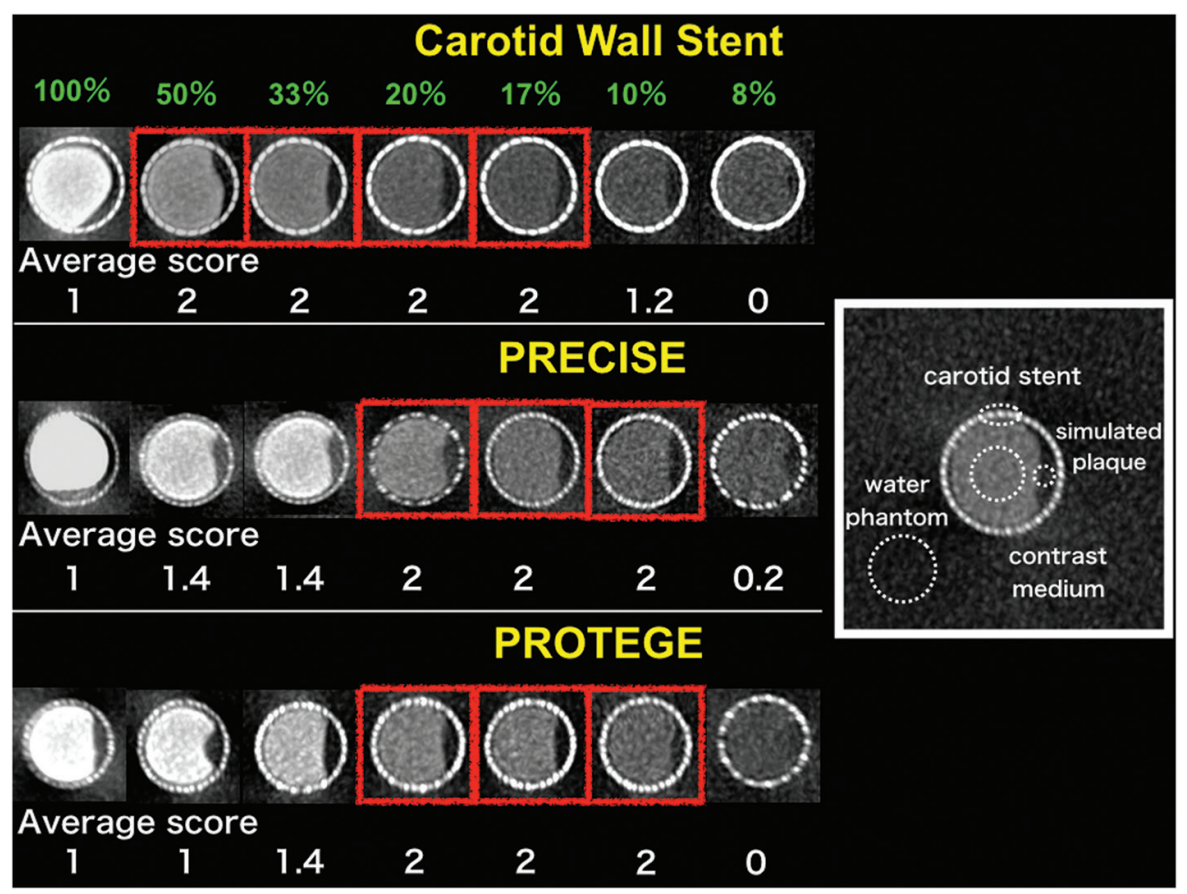

Fig. 2 Phantom images of various carotid artery stents at different contrast dilution ratios. Phantom images of seven simulated vessels prepared by encapsulating $100 \%-8 \%$ diluted contrast medium in three stent types; Carotid Wall Stent (Boston Scientific, Natick, MA, USA), PRECISE (Johnson \& Johnson, Miami, FL, USA), and PROTÉGÉ (Covidien, Irvine, CA, USA), and mean scores of visual evaluation. Arrangement plan of ROI for the measurement of pixel values. ROI: regions of interest

slice thickness was $1 \mathrm{~mm}$. Short-axis images in which the CS and simulated plaque were included in one slice were evaluated to examine whether the shape of the CS, simulated plaque, and simulated vessel lumen of the CS were visualized separately in one image.

Images were scored by visual evaluation, and those in which the CS, simulated vessel lumen, and simulated plaque could all be clearly separated were rated as good (score 2), those in which the contrast medium concentration in the simulated vessel lumen was high, and its border with the CS was unclear but could be clearly separated from the simulated plaque were rated as fair (score 1), and those in which the CS could be visually identified, but the contrast medium concentration in the simulated vessel lumen was low, and the vascular lumen was poorly separated from the simulated plaque were rated as poor (score 0 ). The evaluation was performed by five radiology technologists, and the degree of dilution at which the mean score was 2 (rated good by all five evaluators) was regarded as the effective dilution ratio for each CS.

In addition, regions of interest (ROIs) were set using the image processing software ImageJ as shown in Fig. 2, and pixel values of the diluted contrast medium in each simulated vessel phantom, CS, simulated plaque, and water phantom were determined.

\section{Evaluation of the degree of contrast dilution}

The results of the phantom study indicated that the degree of contrast dilution necessary for delineation of the $\mathrm{CS}$ varies among the CS types. Hosokawa et al. $\left.{ }^{4}\right)$ reported that the optimal contrast medium concentration differed between basic experiments and clinical use of $\mathrm{CBCT}$ and ascribed the difference to the arterial blood flow. The blood flow of the common carotid artery in healthy individuals has been reported to be about $9 \mathrm{~mL} / \mathrm{sec}^{5,6)}$ and since the basic injection rate for 3D-RA at our institution is $4 \mathrm{~mL} / \mathrm{sec}$, the contrast medium is considered to be diluted in the common carotid artery. To fill the common carotid artery with a contrast medium at an effective dilution ratio, it is necessary to calculate the dilution ratio in the common carotid artery from the blood flow and injection rate and determine the dilution ratio of the contrast medium used.

We, therefore, calculated the estimated common carotid artery flow rate $\mathrm{F}_{\text {estimate }}(\mathrm{mL} / \mathrm{sec})$ from the mean blood flow velocity (time-averaged maximum velocity [TAMV]) $(\mathrm{mm} / \mathrm{sec})$ measured by carotid duplex ultrasonography (CUS) performed before or after CAS using the pulsed 
Doppler technique and the common carotid artery diameter $\mathrm{A}_{\text {diameter }}(\mathrm{mm})$ measured by 3D-RA on preoperative angiography and determined the estimated intravascular dilution ratio $\mathrm{C}_{\text {estimate }}(\mathrm{mL} / \mathrm{sec})$ in consideration of the contrast medium injection rate $\mathrm{F}_{\text {injection }}(\mathrm{mL} / \mathrm{sec})$ and contrast medium concentration $\mathrm{C}_{\text {contrast }}(\%)$.

The $\mathrm{F}_{\text {estimate }}$ was calculated using the following equation:

$$
\begin{aligned}
& \mathrm{F}_{\text {estimate }}=\mathrm{TAMV} \times\left(\mathrm{A}_{\text {diameter }} / 2\right)^{2} \times \pi \\
& \mathrm{C}_{\text {estimate }}=1 /\left(\mathrm{F}_{\text {estimate }} / \mathrm{F}_{\text {injection }} / \mathrm{C}_{\text {contrast }}\right) \times 100
\end{aligned}
$$

Using these equations, we retrospectively evaluated whether the estimated intravascular dilution ratio was in agreement with the effective dilution ratio shown by the phantom study and whether the contrast dilution ratio used was effective to accurately visualize the CS, vessel lumen, and plaque. The TAMV used for the calculation must be the blood flow velocity in a state dilated by the CS, the values measured by CUS immediately after CAS were used for 3D-RA at CAS, and those immediately before imaging were used for follow-up 3D-RA. In addition, while the contrast medium injection rate for the common carotid artery is basically $4 \mathrm{~mL} / \mathrm{sec}$ at our institution, an injection rate agreed on by the physician and radiation technologist is selected from a range of $3.5-4.5 \mathrm{~mL} / \mathrm{sec}$ for each patient by examining DSA images and the stenosis rate of the common carotid artery before 3D-RA.

Images were rated in individual patients as good, fair, or poor similarly to the phantom study by a conference of five evaluators.

\section{Subjects}

The subjects were 52 patients in whom the TAMV was measured by CUS before and after 3D-RA performed at CAS or a follow-up after CAS between March 2015 and March 2016 (39 immediately after CAS, 13 at follow-up). They consisted of 44 males and 8 females aged 36-86 $(69.8 \pm 7.1)$ years. The CS used was Carotid Wall Stent in 37, PRECISE in 2, and PROTÉGÉ in 13. This study was carried out with approval by the institutional review board of our institution.

\section{Results}

\section{Results of the phantom study to determine the effective dilution ratio}

When the dilution ratio was $100 \%$, the mean score according to visual evaluation was 1 , and the pixel value was higher in the simulated vessel than in the CS in all three CS types. In images, there was no difference in contrast between the CS and simulated vessel as the simulated vessel showed higher density than the CS with halation around it, and it was difficult to confirm their separation. The mean score of visual evaluation was 2 at dilution ratios of $50 \%-17 \%$ in Carotid Wall Stent and at dilution ratios of $20 \%-10 \%$ in PRECISE and PROTÉGÉ, and the pixel values were also clearly separated among the $\mathrm{CS}$, simulated vessel, and simulated plaque at these dilution ratios. In images, the shape of the CS, simulated plaque, and vascular lumen could be clearly differentiated. The mean score of visual evaluation was 1.2 and 0 for the Carotid Wall Stent at 10 and $8 \%$, respectively; 0.2 for PRECISE at $8 \%$, and 0 for PROTÉGÉ at $8 \%$. The pixel values were close between the simulated vessel and simulated plaque. In images, the contrast medium concentration was low in the vascular lumen, and the border with the simulated plaque was obscure, making their separation difficult (Fig. 2, Table 2).

\section{Results of evaluation of the contrast dilution ratio} Table 3 shows the estimated intravascular dilution ratios in the subjects calculated from the contrast dilution ratio and TAMW measured by CUS for each CS and the results of evaluation of clinical images. 3D-RA images of each CS were obtained at a dilution ratio of $100 \%$ before the beginning of this study to examine the vascular shape after dilatation. 3D-RA using diluted contrast medium was performed for the examination of the CS shape, plaque, and vascular lumen in the CS based on the results of the present phantom study. The contrast dilution ratio in patients treated with Carotid Wall Stent was $100 \%$ or $50 \%$. The estimated intravascular dilution ratio was $42.7 \% \pm 18.3 \%$ at a dilution ratio of $100 \%$ and $19.9 \% \pm 5.4 \%$ at $50 \%$ and was within the range of effective dilution ratio. The results of visual evaluation were good in 13 patients $(87 \%)$ at $100 \%$ and in 20 patients (91\%) at $50 \%$.

In the patients treated with PRECISE, the contrast dilution ratio was $100 \%$ or $20 \%$. At $100 \%$, the estimated intravascular dilution ratio was $26.3 \%$, which deviated to the higher side from the range of effective dilution ratio of $20 \%-10 \%$ for PRECISE. At $20 \%$, it was $11.9 \%$ and fell in the range of effective dilution ratio. The results of visual evaluation were fair at $100 \%$ and good at $20 \%$.

In the patients treated with PROTÉGÉ, the contrast dilution ratio was $100 \%, 67 \%$, or $25 \%$. At $100 \%$, the estimated intravascular dilution ratio was $29.8 \% \pm 9.2 \%$, which was higher than the range of effective dilution ratio 


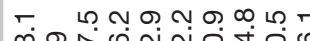

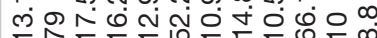

$\therefore+1+1+1+1+1+1+1+1+1+1+1+1$

$\infty \cup 0-10-000$

๒ं

ヘก

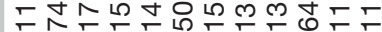

$80+1+1+1+1+1+1+1+1+1+1+1+1$

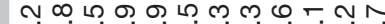
放守穴

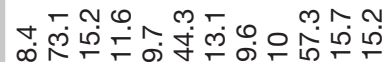
우 $+1+1+1+1+1+1+1+1+1+1+1+1$ -

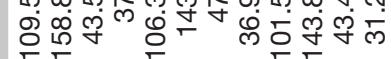

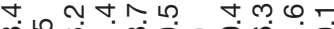
00

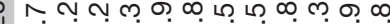
¿

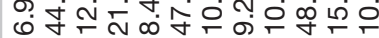
$00+1+1+1+1+1+1+1+1+1+1+1+1$

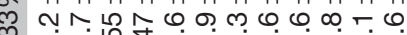
过

ம ํํㅁํ் $00+1+1+1+1+1+1+1+1+1+1+1+1$

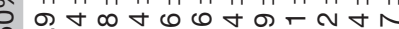

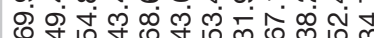

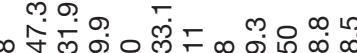

of $20 \%-10 \%$ for PROTÉGÉ. At $67 \%$, it was $14.7 \%$ and was within the range of effective dilution ratio. At $25 \%$, it was $11.5 \% \pm 1.6 \%$ and was within the range of effective dilution ratio in the phantom study. The results of visual evaluation were good in $1(11.1 \%)$ and fair in $8(89 \%)$ at $100 \%$, good in $1(100 \%)$ at $67 \%$, and good in $3(100 \%)$ at 25\% (Table 3).

\section{Clinical Cases}

\section{Carotid Wall Stent}

A 61-year-old male underwent CAS for symptomatic right internal carotid artery stenosis and was found to have restenosis on a follow-up angiography after 2 years. CAS was performed using Carotid Wall Stent (Fig. 3).

At CAS of the right internal carotid artery, the estimated common carotid artery blood flow based on the TAMV measured by CUS was $11.2 \mathrm{~mL} / \mathrm{sec}$, and, as $100 \%$ contrast medium was injected at $4 \mathrm{~mL} / \mathrm{sec}$. The estimated intravascular dilution ratio was $35.7 \%\left(\mathrm{C}_{\text {estimate }}=1 /(11.2 / 4 / 1) \times\right.$ 100). The effective dilution ratio of Carotid Wall Stent was $50 \%-17 \%$, the CS and vascular lumen could be clearly separated in the clinical images obtained, and the absence of plaque protrusion could be confirmed.

At the follow-up, the estimated common carotid artery blood flow based on the TAMV measured by CUS immediately before 3D-RA was $11.8 \mathrm{~mL} / \mathrm{sec}$, and as $50 \%$ dilution of the contrast medium was injected at $4.2 \mathrm{~mL} / \mathrm{sec}$. The estimated intravascular dilution ratio was $17.8 \%$ $\left(\mathrm{C}_{\text {estimate }}=1 /(11.8 / 4.2 / 0.5) \times 100\right)$. In the clinical images obtained, the CS could be clearly separated from the vascular lumen and intimal thickening in the CS.

\section{PROTÉGÉ}

A 66-year-old male underwent bilateral internal carotid endarterectomy for bilateral asymptomatic internal carotid artery stenosis, followed by bilateral CAS. 3D-RA was not performed immediately after CAS of the right internal carotid artery in those days, and it was performed at 1-year and 3-year follow-ups after right CAS and immediately after left CAS. The CS used was PROTÉGÉ on both sides (Fig. 4).

At 1-year follow-up, the estimated common carotid artery blood flow based on the TAMV measured by CUS after CAS was $8 \mathrm{~mL} / \mathrm{sec}$, and as $100 \%$ contrast medium was injected at $3.8 \mathrm{~mL} / \mathrm{sec}$. The estimated intravascular dilution ratio was $47.5 \%\left(\mathrm{C}_{\text {estimate }}=1 /(8 / 3.8 / 1) \times 100\right)$. The effective dilution ratio of PROTÉGÉ was $20 \%-10 \%$, and, in the clinical images obtained, the density of the vascular 
Table 3 Results of calculation of the estimated intravascular dilution ratio and visual evaluation in individual CAS cases

\begin{tabular}{lcccccc} 
& $\mathrm{C}_{\text {contrast }}(\%)$ & $\mathrm{F}_{\text {estimate }}$ & $\mathrm{C}_{\text {estimate }}(\%)$ & \multicolumn{3}{c}{ Visual evaluation (cases) } \\
Carotid Wall Stent & $100 \%(\mathrm{~mL}=15)$ & $12.4 \pm 5.7$ & $42.7 \pm 18.3$ & 13 & 2 & 0 \\
$(\mathrm{n}=37)$ & $50 \%(\mathrm{n}=22)$ & $11.8 \pm 3.2$ & $19.9 \pm 5.4$ & 20 & 0 & 2 \\
PRECISE & $100 \%(\mathrm{n}=1)$ & 15.2 & 26.3 & 0 & 1 & 0 \\
$(\mathrm{n}=2)$ & $20 \%(\mathrm{n}=1)$ & 7 & 11.9 & 1 & 0 & 0 \\
PROTÉGÉ & $100 \%(\mathrm{n}=9)$ & $14.5 \pm 4.5$ & $29.8 \pm 9.2$ & 1 & 8 & 0 \\
$(\mathrm{n}=13)$ & $67 \%(\mathrm{n}=1)$ & 18.1 & 14.7 & 1 & 0 & 0 \\
& $25 \%(\mathrm{n}=3)$ & $11.8 \pm 3.2$ & $11.5 \pm 1.6$ & 3 & 0 & 0
\end{tabular}

Values are mean \pm standard deviation. CAS: carotid artery stenting; $\mathrm{C}_{\text {contrast }}$ : concentration of contrast medium; $F_{\text {estimate: }}$ : estimated carotid arterial blood flow; $\mathrm{C}_{\text {estimate }}$ : estimated intravascular contrast agent concentration

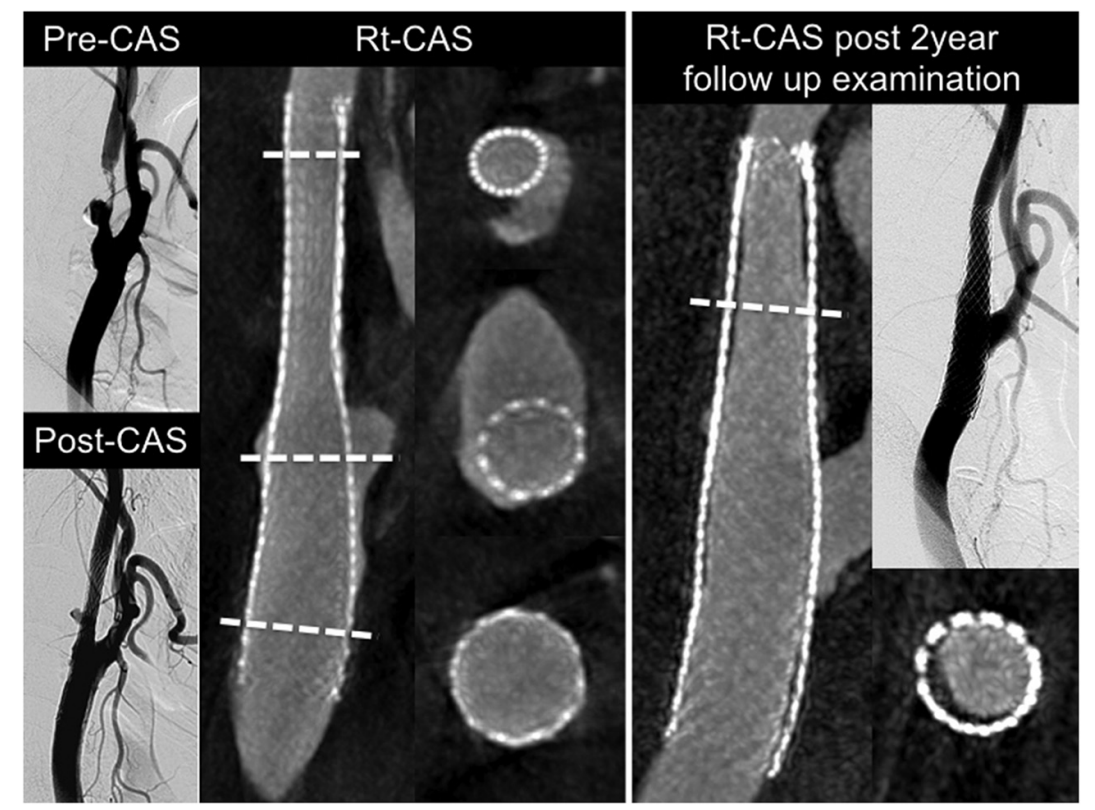

Fig. 3 DSA and 3D-RA images of CS (Carotid Wall Stent) in the same patient. Left: 3D-RA at CAS of the right common carotid artery; $100 \%$ contrast medium was used. Right: 3D-RA at follow-up 2 years after CAS; $50 \%$ diluted contrast medium was used. 3D-RA: 3D-rotational angiography; CS: carotid stent; CAS: carotid artery stenting

lumen was higher than that of the CS, and the plaque could be clearly separated, but separation between the CS and vascular lumen was unclear.

At 3-year follow-up, the estimated common carotid artery blood flow based on the TAMV measured by CUS immediately before 3D-RA was $9.1 \mathrm{~mL} / \mathrm{sec}$, and as a $25 \%$ dilution of the contrast medium was injected at $4 \mathrm{~mL} / \mathrm{sec}$. The estimated intravascular dilution ratio was $11 \%\left(\mathrm{C}_{\text {estimate }}\right.$ $=1 /(9.1 / 4 / 0.25) \times 100)$. In the clinical images obtained, there was a density difference between the CS and vascular lumen, and the CS, vascular lumen, and intimal thickening, which were increased, compared with previous images, were clearly distinguished.

At the placement of $\mathrm{CS}$ in the left internal carotid artery, the estimated common carotid artery blood flow based on the TAMV measured by CUS immediately before CAS was $18.1 \mathrm{~mL} / \mathrm{sec}$, and as it was faster than the mean blood flow of the right internal carotid artery, a $67 \%$ dilution of the contrast medium was injected at $4 \mathrm{~mL} / \mathrm{sec}$. The estimated intravascular dilution ratio was $14.8 \%\left(\mathrm{C}_{\text {estimate }}=\right.$ $1 /(18.1 / 4 / 0.67) \times 100)$. In the clinical images obtained, there was a density difference between the CS and vascular lumen, and clear separation between the CS and vascular lumen and the absence of plaque protrusion in the CS could be confirmed.

\section{PRECISE}

A 76-year-old male underwent CAS for asymptomatic right internal carotid artery stenosis and showed restenosis on a follow-up after 2 years. The CS used was PRECISE (Fig. 5). 


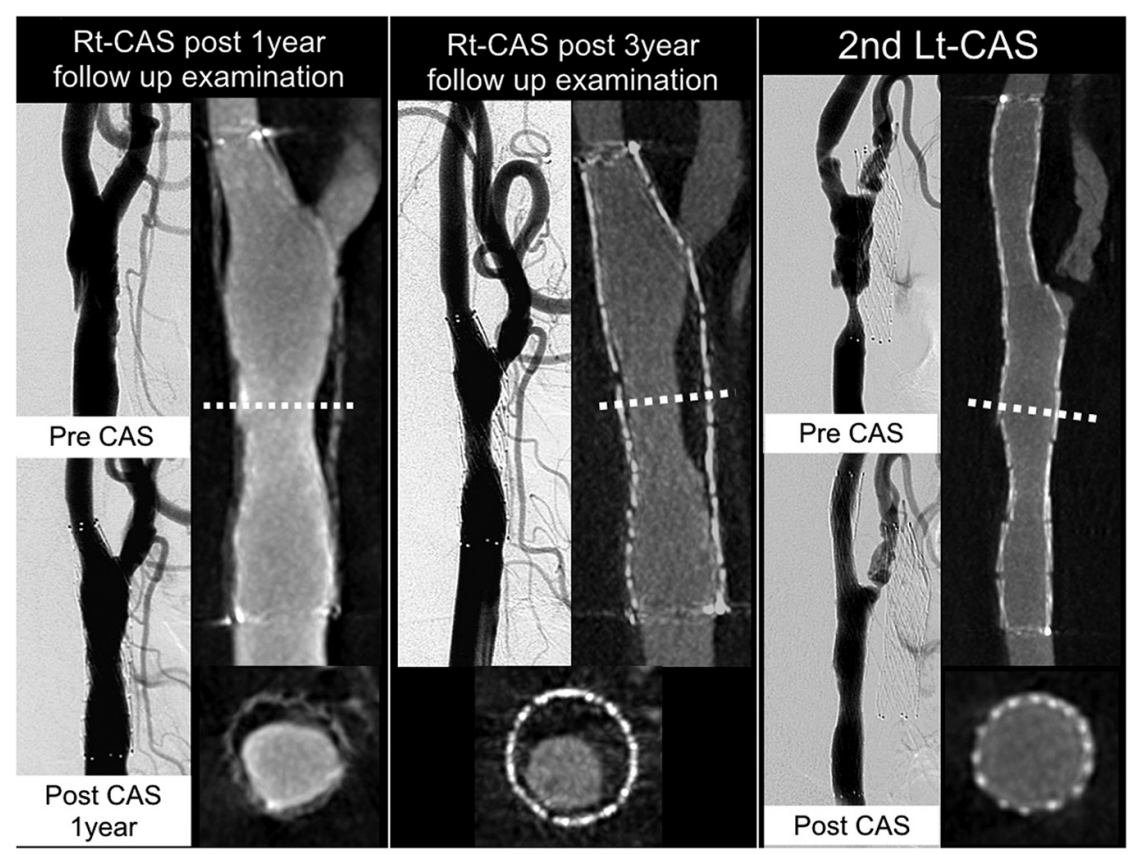

Fig. 4 DSA and 3D-RA images of CS (PROTÉGÉ) in the same patient. Left: 3D-RA at follow-up 1 year after CAS of the right common carotid artery; $100 \%$ contrast medium was used. Middle: 3D-RA at follow-up 3 years after CAS; $25 \%$ contrast medium was used. Right: 3D-RA at CAS of the left common carotid artery; $67 \%$ contrast medium was used. CAS: carotid artery stenting; CS: carotid stent; 3D-RA: 3D-rotational angiography

The estimated common carotid artery blood flow at CAS was $15.2 \mathrm{~mL} / \mathrm{sec}$ based on the TAMV measured by CUS after CAS. As $100 \%$ contrast medium was injected at $4 \mathrm{~mL} / \mathrm{sec}$, the estimated intravascular dilution ratio was $26.3 \%\left(\mathrm{C}_{\text {estimate }}=1 /(15.2 / 4 / 1) \times 100\right)$. Since the effective dilution ratio of PRECISE was $20 \%-10 \%$, the density in the clinical images obtained was similar between the CS and vascular lumen, and the separation between the CS and vascular lumen was unclear.

On 2-year follow-up angiography, the estimated common carotid artery blood flow based on the TAMV measured by CUS immediately before the examination was $7 \mathrm{~mL} / \mathrm{sec}$, and a $20 \%$ dilution of the contrast medium was injected at $4.2 \mathrm{~mL} / \mathrm{sec}$ to adjust the estimated intravascular dilution ratio to $12 \%$ according to the results of this study. Thus, the estimated intravascular dilution ratio was $12 \%$ $\left(\mathrm{C}_{\text {estimate }}=1 /(7 / 4.2 / 0.2) \times 100\right)$. In the clinical images obtained, the CS, vascular lumen, and restenosis due to intimal thickening could be clearly separated from one another.

\section{Discussion}

Evaluation after CAS is performed primarily by noninvasive CUS, and detailed examination by angiography is made when stenosis is suggested. In DSA, the degree of dilatation after CS placement and protrusion of thrombus and atheroma in the CS are examined from one direction or from two directions using a biplane system, and a large number of scans are necessary to obtain data from many directions. IVUS, by which the properties and shape of the lesion, degree of stenosis, and state in the CS can be evaluated, provides information that complements DSA. However, as a device must be passed inside the CS, there is a considerable risk of perforation and cerebral infarction, and embolic protection devices are necessary for their prevention. ${ }^{78)}$ Evaluation by IVUS can be implemented safely immediately after stenting because embolic protection devices may be placed in the vessels, but embolic protection devices are not used in diagnostic angiography for the follow-up after CAS, and imaging tools such as DSA and 3D-RA are used. Advantages of using 3D-RA for the evaluation after CAS are that no device other than the catheters used for angiography is necessary and that no special skill is required except for the use of diluted contrast medium. Therefore, IVUS is selected when embolic protection devices are placed, but 3D-RA is effective for diagnostic angiography not using an embolic protection device.

Both 3D-RA and HRCBCT have outstanding ability to visualize the density difference produced by a contrast medium. Therefore, they are excellent in presenting volume 


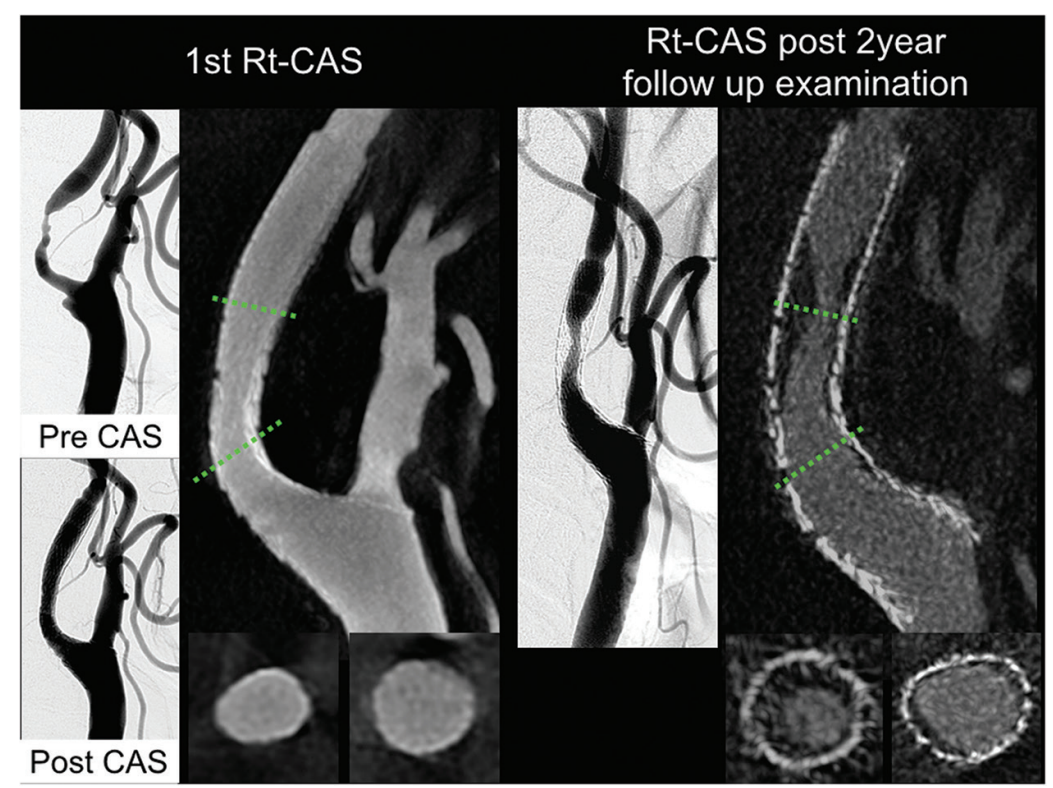

Fig. 5 DSA and 3D-RA images of CS (PRECISE) in the same patient. Left: 3D-RA at CAS of the right common carotid artery; $100 \%$ contrast medium was used. Right: 3D-RA at follow-up 2 years after CAS; $20 \%$ diluted contrast medium was used. CAS: carotid artery stenting; CS: carotid stent; 3D-RA: 3D-rotational angiography

rendering and multi-planar images by high-resolution reconstruction. Since the look up table, which shows the contrast of HRCBCT images, is linear, the overall density is emphasized. Also, the number of acquired images during a scanning time of 20 seconds was 620 , and this large number of images acquired provide excellent low-contrast resolution, which is advantageous for visualization of vessels with poor contrast enhancement such as peripheral vessels and veins. However, it causes contrast enhancement of not only blood vessels, but also artifacts such as metals and pulses. Conversely, as the look up table of 3D-RA is $\mathrm{S}$-shaped, contrast can be enhanced in an optional concentration range, and the technique has excellent high-contrast resolution, which is advantageous for visualization of objects with density differences such as the blood vessel and metals including the CS. In 3D-RA, metal artifacts are smaller than in HRCBCT because of automatic changes of the tube voltage. The number of images obtained in a scanning time of 4 seconds is 120 , and while the technique is inappropriate for imaging of objects that require lowcontrast resolution, it is less likely to be affected by artifacts due to metals and pulses. In this study, 3D-RA was considered appropriate because we intended imaging of both the stent and the contrasted vessel and high-speed imaging to minimize the effects of movements due to neck pulsation.

As shown in the basic experiment, a diluted contrast medium with a high concentration not exceeding the density of the CS is necessary to obtain contrast between the CS and vascular lumen. Elgiloy, which is the material of Carotid Wall Stent, shows high X-ray absorption. It has a dense closed-cell-type stent structure. On the other hand, PRECISE and PROTÉGÉ are made of nitinol, which shows lower X-ray absorption than Elgiloy. They are open-cell-type stents with wide free cell areas. Since they are made of a material with lower X-ray absorption compared with Carotid Wall Stent and have a narrower total area, a diluted contrast medium at a lower concentration is considered to be more appropriate for their visualization than Carotid Wall Stent.

Hosokawa et al. ${ }^{4)}$ reported that a $5 \%$ diluted contrast medium was optimal in a basic experiment for both Carotid Wall Stent and PRECISE, but we set the concentration at $50 \%-17 \%$ for Carotid Wall Stent and $20 \%-10 \%$ for PRECISE. While these concentration differences are considered to be explained partly by the differences in the look up table specific to the device and number of images acquired but, importantly, also by the shape and material of the CS. Therefore, we consider it desirable to alter the concentration of the contrast medium for different CS types.

In the clinical evaluation, we determined the dilution ratio of the contrast medium according to the TAMV measured by CUS, but as the TAMV varied among the 52 patients, it was difficult to apply the same dilution ratio to all patients. For satisfactory imaging, it is important to 
uniformly fill the common carotid artery with a contrast medium, and its concentration must be adjusted according to the vascular diameter and shape of stenosis. As the injection rate of the contrast medium may surpass the blood flow velocity, particularly, in patients with severe stenosis, there is a possibility of replacement by the contrast medium without dilution by the blood flow of the common carotid artery. Therefore, an injection rate appropriate for the blood flow velocity and a low-concentration contrast medium are recommended for patients with severe stenosis because a contrast medium with a larger specific gravity than blood is likely to pool.

While it is possible to determine the appropriate dilution ratio of the contrast medium by expecting the blood flow before CAS or angiography, the timing of the measurement of the TAMV of the carotid artery is considered to be a problem. The TAMV determined by CUS before angiography can be used for follow-up examination because there is no change in the blood flow velocity during the examination. In CAS, however, the blood flow velocity after CS deployment is necessary as the blood flow changes after carotid artery dilatation. For easy intraoperative determination of the TAMV, measurement immediately before 3D-RA using a portable echograph is considered useful. Jo et al. ${ }^{9)}$ reported a method to dilute the contrast medium according to the blood flow of the internal carotid artery based on the measurement using transcranial Doppler (TCD). Since this technique is designed for the internal carotid artery distal to the catheter, it cannot be used for visualization of the common carotid artery. However, TCD is used at our institution for real-time measurement of the intracranial blood flow velocity during CAS and prediction of cerebral infarction, and the device itself has no effect on the images such as its overlapping in the images. If the blood flow velocity of the common carotid artery can be converted using TCD, the technique is expected to be very effective for ensuring the safety and improving the throughput, but this theme is left to future studies.

\section{Conclusion}

For the evaluation of the vascular lumen after CAS, it is essential to visualize the CS, plaque and intimal thickening, and vascular lumen in the stent. Using the technique described in this report, it is possible to optimize the dilution ratio of the contrast medium for 3D-RA according to the CS type and individual difference in the carotid artery blood flow.

\section{Acknowledgments}

The authors express our sincere gratitude to the staff of the Department of Neuroendovascular Therapy and members of the Department of Radiology, Toranomon Hospital for their cooperation in this study.

\section{Disclosure Statement}

Neither the first author nor any of the coauthors have any conflicts of interest.

\section{References}

1) Snoeren RM, Söderman M, Kroon JN, et al: High-resolution 3D X-ray imaging of intracranial nitinol stents. Neuroradiology 2012; 54: 155-162.

2) Patel NV, Gounis MJ, Wakhloo AK, et al: Contrastenhanced angiographic cone-beam CT of cerebrovascular stents: experimental optimization and clinical application. AJNR Am J Neuroradiol 2011; 32: 137-144.

3) Benndorf G, Strother CM, Claus B, et al: Angiographic CT in cerebrovascular stenting. AJNR Am J Neuroradiol 2005; 26: 1813-1818.

4) Hosokawa S, Kawai N, Sato M, et al: Optimal contrast material concentration for distinguishing among carotid artery lumen, carotid stent, and neck in cone-beam computed tomography during carotid angiography: basic and clinical studies. Jpn J Radiol 2012; 30: 358-364.

5) Wada T, Kodaira K, Fujishiro K, et al: Correlation of common carotid flow volume measured by ultrasonic quantitative flowmeter with pathological findings. Stroke 1991; 22: 319-323.

6) Takahashi H, Konno S, Watanabe K, et al: [Effects of aging on blood flow volumes, velocities, mean vessel diameters, and vessel elasticities of the common carotid artery in healthy voluteers: a longitudinal study]. Jpn J Stroke 1997; 19: 301-307. (in Japanese)

7) Musialek P, Pieniazek P, Tracz W, et al: Safety of embolic protection device-assisted and unprotected intravascular ultrasound in evaluating carotid artery atherosclerotic lesions. Med Sci Monit 2012; 18: MT7-MT18.

8) Tsurumi A, Miyachi S, Yoshida J: [The role of IVUS virtual histology in cerebrovascular disorders carotid artery stenting]. Currently Practical Neurosurgery 2007; 17: 446-454. (in Japanese)

9) Jo KI, Kim SR, Choi JH, et al: Contrast-enhanced angiographic cone-beam computed tomography without pre-diluted contrast medium. Neuroradiology 2015; 57: 1121-1126. 